\title{
Improvement of pyridoxine-induced peripheral neuropathy by Cichorium intybus hydroalcoholic extract through GABAergic system
}

\author{
Farkhonde Hasannejad $^{1} \cdot$ Malek Moein Ansar ${ }^{2,3} \cdot$ Mohammad Rostampour $^{2,4} \cdot$ Edris Mahdavi Fikijivar $^{5}$. \\ Behrooz Khakpour Taleghani ${ }^{2,4}$ (D)
}

Received: 21 March 2018 / Accepted: 5 January 2019 / Published online: 2 February 2019

(C) The Physiological Society of Japan and Springer Japan KK, part of Springer Nature 2019

\begin{abstract}
Pyridoxine (vitamin B6) toxicity is a well-known model for peripheral neuropathy. GABA and glutamate are two neurotransmitters in neural pathways involved in the peripheral neuropathy. Cichorium intybus (Chicory) contains glycosides and triterpenoids, which inhibit glutamatergic transmission and enhance GABAergic transmission. The present study was aimed at studying the effect of chicory extract (CE) on the pyridoxine-induced peripheral neuropathy with a particular focus on glutamatergic and GABAergic systems. In this experimental study, a high dose of pyridoxine ( $800 \mathrm{mg} / \mathrm{kg}$, i.p.) was injected for 14 days to induce neuropathy in male rats. To evaluate the behavioral symptoms, three tests including rotarod, hot plate, and foot fault were used. After the induction of neuropathy, CE $(50 \mathrm{mg} / \mathrm{kg}$ i.p.) was injected intraperitoneally for 10 consecutive days. Morphologically, the sciatic nerve and the DRG neurons were evaluated in the control, neuropathy, and chicory groups by H\&E staining. For evaluating the mechanism, picrotoxin $(1 \mathrm{mg} / \mathrm{kg})$ and MK-801 $(0.1 \mathrm{mg} / \mathrm{kg}) \mathrm{were}$ also individually injected $15 \mathrm{~min}$ before the extract administration. The concentration of TNF- $\alpha$ in rat sciatic nerve and DRG neurons were also measured by enzyme-linked-immunoassay (ELISA). Morphological and physiological changes occurred in the DRG and sciatic nerve following pyridoxine intoxication. The CE exerted an anti-neuropathic effect on the sciatic nerve and DRG neurons and also decreased reaction time in hot plate test $(p<0.05)$, increased balance time in rotarod test $(p<0.001)$, and improved foot fault performance $(p<0.01)$. Moreover, CE administration reduced TNF- $\alpha$ level in DRG $(p<0.001)$ and sciatica nerve $(p<0.001)$. Picrotoxin, unlike MK-801, showed a significant difference in all three behavioral tests and reduced TNF- $\alpha$ content in comparison with group received extraction alone (with $p<0.001$ for all three tests). Our results showed beneficial effects of $\mathrm{CE}$ on pyridoxine-induced peripheral neuropathy. Modulating of the GABAergic system mediated by TNF- $\alpha$ may be involved in the anti-neurotoxic effect of CE.
\end{abstract}

Keywords Peripheral neuropathy $\cdot$ Pyridoxine $\cdot$ DRG $\cdot$ Sciatic nerve $\cdot$ CE $\cdot$ GABA $\cdot$ Glutamate

Behrooz Khakpour Taleghani

khakpourtaleghani.behrooz@gmail.com

Farkhonde Hasannejad

93it.hasannejad@gmail.com

Malek Moein Ansar

malekmoein.ansar@gmail.com

Mohammad Rostampour

rost_v@yahoo.com

Edris Mahdavi Fikijivar

mahdavi.edris@gmail.com
1 School of Medicine, Guilan University of Medical Sciences, Rasht, Iran

2 Neuroscience Research Center, School of Medicine, Guilan University of Medical Sciences, Rasht, Iran

3 Department of Biochemistry, School of Medicine, Guilan University of Medical Sciences, Rasht, Iran

4 Department of Physiology, School of Medicine, Guilan University of Medical Sciences, Rasht, Iran

5 Guilan University of Medical Sciences, Rasht, Iran 


\section{Introduction}

Neuropathy means the two-way transmission disruptions between either the brain or the spinal cord and different parts of the body [1]. Central and peripheral nerve damage can be caused by neuropathy [2]. In the early stages of neuropathy, progressive symptoms such as the loss of sensation and pain in distal limbs can be observed. Over time, numbness may be extended to proximal organs. Major causes of neuropathy include diabetes, genetic disorders, and drug toxicity [3]. The prevalence of neuropathy in the community is $2.4 \%$, but increases with age to $8 \%$ [4].

Vitamin B6 has been called pyridoxine because of its similar structure to pyridine [5]. Vitamin B6 is watersoluble and naturally exists in three forms: pyridoxine (pyridoxol, the alcohol), pyridoxamine (the amine), and pyridoxal (the aldehyde) [6]. Although the family of B vitamins are often non-toxic, high doses of pyridoxine cause peripheral sensory nerve damage [7]. Studies on human [8] and rat [9] have shown the effect of pyridoxine toxicity on the soma of dorsal root ganglion (DRG) neurons causing leading to necrosis and broad damage to long myelinated fibers and eventually to cell death [10]. Oxidative stress plays an important role in nerve damage as well as a variety of peripheral neuropathies including oxaliplatin chemotherapy and diabetes-induced neuropathy [11-13]. Furthermore, pre-inflammatory cytokines and the cyclooxygenase-2 (COX2) enzyme, as important inflammatory mediators, may increase various types of nerve damage and neuropathy [14]. Meanwhile, there are free radicals produced by the function of $\mathrm{COX}-2$, which not only augment the oxidative stress but also induce apoptosis in GABAergic neurons [15]. GABA, as an inhibitory neurotransmitter, is synthesized within the presynaptic neurons and is kept in synaptic vesicles. There are two classes of GABA receptors: fast-acting inotropic $\mathrm{GABA}_{\mathrm{A}}$ and slower-acting metabotropic $\mathrm{GABA}_{\mathrm{B}}$ receptor [16]. According to the research, dorsal horn of the spinal cord and sciatic nerve injury lessens the expression levels of GABA and its receptors [17]. Glutamate, as an excitatory transmitter, on the one hand, increases mGluR5 receptor existing in the DRG and enhances release of inflammatory components [18] and on the other hand, elevates oxidative stress and apoptosis in cerebral vascular endothelial cells [19].

Cichorium intybus, known as "Chicory" belongs to the Asteraceae family, and is widespread in Asia and Europe [20]. All parts of the plant contain medicinal compounds such as alkaloids, vitamins, chlorophyll pigments, flavonoids, a sesquiterpene lactone, glycosides, triterpenoids, caffeic acid derivatives, saponins, and tannins [21, 22]. According to previous studies, chicory extract (CE) has the potential to reduce oxidative stress agents and has been used as an anti-diabetic, anti-hepatotoxic, and antiinflammatory agent for the treatment of various ailments [23-25].

Due to the presence of various medicinal compounds in $\mathrm{CE}$ and its probable role in control of inflammatory processes and also the possible interference of GABAergic and glutamatergic systems in nerve injury and neuropathy, we investigated the therapeutic effect of hydroalcoholic chicory root extract on pyridoxine-induced neuropathy and also examined the possible role of GABAergic and glutamatergic systems as the candidate mechanisms of CE action.

\section{Materials and methods}

\section{Preparation of CE}

The roots of chicory were collected from highland rural areas around Guilan Province, Iran. The roots washed and dried in the natural condition at $24-26{ }^{\circ} \mathrm{C}$ for 3-4 days. Then, $100 \mathrm{~g}$ of the chicory powder was soaked in $300 \mathrm{cc}$ methanol hydroalcoholic solution (800 cc methanol 96\% with $200 \mathrm{cc}$ water) and was extracted with a Soxhlet extractor. The resulting extract was dried in dry heat at $40-50{ }^{\circ} \mathrm{C}$ for 3-4 days. The dried extract was dissolved in saline and applied to the treatment group.

\section{GC-MS analysis}

All analysis was carried out on a GC-MS system from Agilent Technologies (Wilmington, DE, USA). The GC-MS system used for this study consisted of a model 7890A gas chromatograph, a model 5970B mass selective detector, and electron ionization (EI) mode $(70 \mathrm{eV})$. A fused-silica capillary column (30 $\mathrm{m} \times 250 \mathrm{~m}$ i.d., $0.25-\mathrm{m}$ film thickness) from Scitech Scientific was also used. The temperature program of GC was as follows: initial temperature was $120^{\circ} \mathrm{C}$, held for $2 \mathrm{~min}$, increased to $140{ }^{\circ} \mathrm{C}$ at a rate of $2{ }^{\circ} \mathrm{C} / \mathrm{min}$, then to $220^{\circ} \mathrm{C}$ at a rate of $3{ }^{\circ} \mathrm{C} / \mathrm{min}$, and finally to $320^{\circ} \mathrm{C}$ at a rate of $7^{\circ} \mathrm{C} / \mathrm{min}$ and held for $15 \mathrm{~min}$. The split ratio was $1: 12$, injection temperature was $290{ }^{\circ} \mathrm{C}$, transfer line temperature was $310^{\circ} \mathrm{C}$, and ion source temperature was $240{ }^{\circ} \mathrm{C}$. A quadruple mass spectrometer (MS $5975 \mathrm{C}$ inrtXL EI/CI MSD with triple-axis detector) was operated at $70 \mathrm{eV}$ in the electron impact CTC PAL auto sampler.

\section{Identification of phytocomponents}

The interpretation of the mass spectrum GC-MS was carried out using the database of National Institute Standard and Technology (NIST). The various compounds with their different retention indices and area percentage were ascertained 
using NIST Ver. 2.1 MS data library [26]. For quantitative analysis, the concentrations of compounds (\%) were calculated by combining their corresponding chromatographic peak area.

\section{Animals}

In this study, 56 male Wistar rats with an average body weight of 200-250 g were used. Animals were maintained (four per cage) under a standard condition with ambient temperature $\left(22 \pm 2{ }^{\circ} \mathrm{C}\right)$ and reverse light-dark cycle $(12 / 12 \mathrm{~h})$ with food and water available ad libitum.

\section{Determination of the effective dose of chicory root extract}

In the pilot experiments, a lethal dose (LD50) of CE was determined by injection of different extract doses $(100,200$, 500,1000 , and $2000 \mathrm{mg} / \mathrm{kg} / \mathrm{i}$. p.) for 10 days and the mortality rate of the animals was observed. Eventually, LD50 was selected $(250 \mathrm{mg} / \mathrm{kg} /$ i.p. $)$ in the chronic administration by the log dose probit analysis. Then, 5,10 , and $20 \%$ of LD50 was injected (i.p.) to neuropathic rats and the effective dose was selected consequently. In our study, $20 \%$ of the lethal dose $(50 \mathrm{mg} / \mathrm{kg})$ had improving effect and decreased neuropathic signs in all behavioral tests.

\section{Induction of pyridoxine neuropathy in rats}

Pyridoxine (Osveh, Tehran, Iran) was dissolved in sodium chloride $0.9 \%$ solution at $50{ }^{\circ} \mathrm{C}$ and injected intraperitoneally $(800 \mathrm{mg} / \mathrm{kg} / \mathrm{ml})$. The injections were carried out at about 9:00 am for 14 consecutive days [27].

\section{Drug administration}

After induction of neuropathy, CE (50 mg/kg/i.p.) as the therapeutic dose was injected for 10 days. To investigate the role of GABAergic and Glutamatergic systems, picrotoxin (GABA antagonist, Sigma-Aldrich, St. Louis, MO, USA) and MK-801 (glutamate antagonist, Sigma-Aldrich, St. Louis, MO, USA) were used. The drugs-picrotoxin ( $1 \mathrm{mg} /$ kg/i.p.) and MK-801 (0.1 mg/kg/i.p.) —were injected $15 \mathrm{~min}$ before the administration of CE.

\section{Experimental groups}

The rats were divided into six groups $(N=7)$ : (1.) Control: received normal saline (NS), (2.) NP: received $800 \mathrm{mg} / \mathrm{kg}$ pyridoxine, (3.) $\mathrm{NP}+\mathrm{NS}$, (4.) $\mathrm{NP}+\mathrm{CE}(50 \mathrm{mg} / \mathrm{kg} / \mathrm{i} . \mathrm{p})$, (5.) $\mathrm{NP}+$ picrotoxin $(1 \mathrm{mg} / \mathrm{kg})+\mathrm{CE}$, and (6.) NP + MK-801 $(0.1 \mathrm{mg} / \mathrm{kg})+$ CE.

\section{Behavioral tests}

\section{Rotarod test}

The animals balance time was measured by rotarod. The rotarod apparatus includes some automated rods with $5 \mathrm{~cm}$ diameter and 12 rotations per min. Afterwards, the animal balance time was recorded (maximum $180 \mathrm{~s}$ ). This experiment was carried out three times and the mean time was measured.

\section{Hot plate test}

The rats were put on a hot plate at a temperature of $55^{\circ} \mathrm{C}$. Meanwhile, the animal behaviors such as raising one's paw, licking one's foot or jumping to escape from the heat (the reaction time) were recorded.

\section{Foot fault counting test}

The apparatus consisted of small bars with $1.5 \mathrm{~cm}$ far from each other and 1-m long was laid above a surface. The rat was placed at one end and its balance along the way was monitored. Along the movement, due to the weight-bearing of paws, falling or slipping might happen. Then, the number of errors in the animal's feet during walking was counted. At the end, each error was taken into consideration when the rat could not pass through the grid and its paws were trapped between the bars.

\section{Histopathological evaluation}

For evaluation of neuropathy, the animals in the control and neuropathy group, after 14 days, and then the chicory group, 10 days later, were sacrificed. In all animals, part of the sciatic nerve and lumbar dorsal root ganglia in the L4-L6 area were taken for histological analysis (Fig. 1). After preparation of tissues for hematoxylin and eosin (H\&E) staining, DRG was sectioned transversely and the sciatic nerve was sectioned both transversely and longitudinally $(4-6 \mu \mathrm{m})$. For exposure of the nucleus and the cytoplasm, sections were stained with hematoxylin and then eosin (Sigma-Aldrich, St. Louis, MO, USA). The H\&E-stained sections were used for microscopic analysis (inverted fluorescence microscope, Olympus, Tokyo, Japan).

\section{Determination of tumor necrosis factor-a in DRG and sciatic nerve}

In our experimental groups, DRG and sciatic nerve in the control and neuropathy group, after 14 days and the CE, $\mathrm{CE}+$ picrotoxin and $\mathrm{CE}+\mathrm{MK}-801$ treatment groups 10 days later, were removed $(N=5)$. Tissues were homogenized and 
Fig. 1 Dorsal of the lumbar segment contains DRG neurons (a) and site of the sciatic nerve (b)
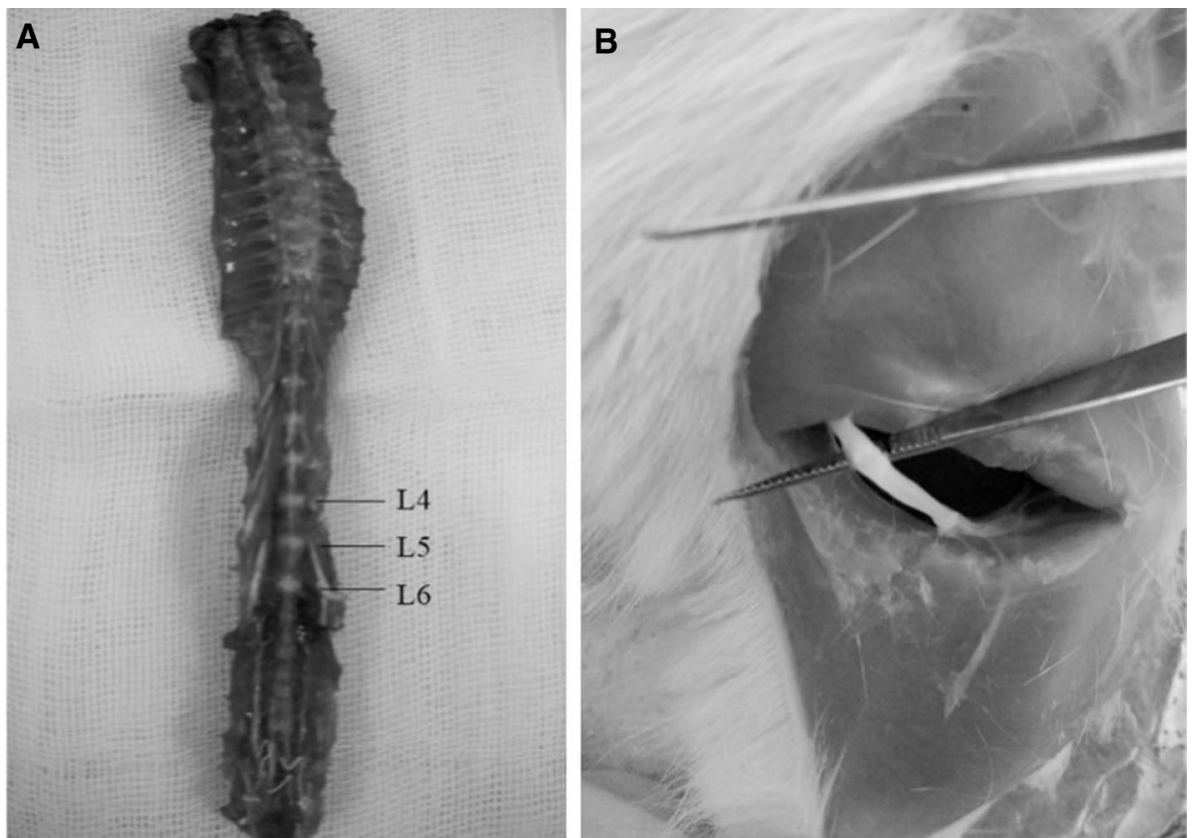

centrifuged at $15,000 \times g$ for $10 \mathrm{~min}$ at $4{ }^{\circ} \mathrm{C}$. The supernatant was collected and analyzed for TNF- $\alpha$ using the Rat TNF- $\alpha$ ELISA Kit (MyBioSource) according to the manufacturer's instructions.

\section{Statistics}

To investigate the normality of the data, the Shapiro test was used. A global analysis of the data was performed using onefactor or repeated-measures analysis of variance (ANOVA); one- and two-way ANOVA tests were used. The level of significance was set at $p<0.05$ [28].

\section{Result}

\section{GC-MS (gas chromatography-mass spectrometry) analysis}

The results pertaining to GC-MS analysis of the chicory methanolic extract led to the identification of a number of compounds. These compounds were identified through mass spectrometry attached with GC. The various chemical constituents identified in chicory root extract are shown in Table 1 and Fig. 2.

\section{Pyridoxine toxicity and induction of peripheral neuropathy}

No toxicity symptoms were observed during the first week but all the animals showed severe signs of neuropathy from the initiation of the second week. Functional defects
Table 1 Compounds identified in the methanolic extract of chicory root by GC-MS

\begin{tabular}{|c|c|c|}
\hline Name of the compound & Retention time & Peak area $\%$ \\
\hline Octadecatrienoic acid & 10.123 & 5.62 \\
\hline Tetrahydrobenzene (isoquinoline) & 13.245 & 1.91 \\
\hline Catechins (flavonoid) ${ }^{\mathrm{a}}$ & 16.325 & 6.56 \\
\hline Quercetin (flavonoid) ${ }^{\mathrm{a}}$ & 20.369 & 5.81 \\
\hline Hexadecanoic acid & 23.047 & 25.03 \\
\hline Diol acetate & 26.82 & 4.52 \\
\hline Cyclolanost & 30.874 & 1.11 \\
\hline 8-Deoxylactucin (sesquiterpene) ${ }^{\mathrm{a}}$ & 33.61 & 17.15 \\
\hline Lactucopicrin (sesquiterpene) ${ }^{\mathrm{a}}$ & 37.321 & 12.08 \\
\hline Epoxy naphtha & 40.497 & 4.03 \\
\hline Hexadecene & 44.125 & 1.1 \\
\hline Cyanidin (glycoside) ${ }^{\mathrm{a}}$ & 47.569 & 7.32 \\
\hline Delphinidin $(\text { glycoside })^{\mathrm{a}}$ & 50.048 & 6.54 \\
\hline Lupeol (terpenoid) ${ }^{\mathrm{a}}$ & 54.71 & 1.22 \\
\hline
\end{tabular}

${ }^{\mathrm{a}}$ These compounds have potential effects on the GABA receptor

appeared gradually and the rats were deprived of the ability to move. Consequently, it was the function of the hind paws that was decreased earlier than the other limbs and led the animals to drag their body in order to move. So, the rats were unable to coordinate all their four limbs and were suffering from motor and sensory ataxia. Moreover, according to Fig. 3, the rats were unable to either walk or stand normally on all their four paws and moved by pushing their bodies utilizing their tails as well as the pressure of the underside. 


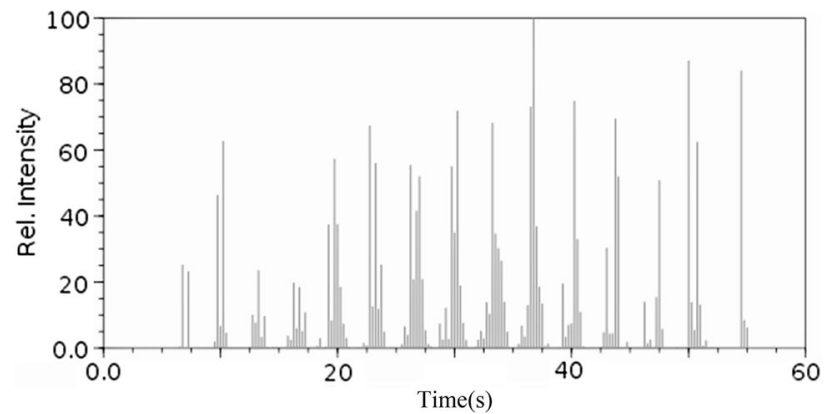

Fig. 2 GC-MS chromatogram of the methanolic extract of chicory root

\section{Rotarod test}

Statistically, according to Fig. 4a, the injection of pyridoxine $800 \mathrm{mg} / \mathrm{kg} / \mathrm{i} . \mathrm{p}$. decreased the animal balance time on the rotarod significantly in comparison with the control group $(138.66 \pm 3.76$ vs. $4.17 \pm 1.64, p<0.001)$. The pyridoxinetreated group was unable to remain on the rotarod and the rats fell down the platform in a short time but the control group could easily move on the rotarod. Conducting the
CE treatment, the animal balance time on the rotarod, compared with the saline group, showed a significant increase $(24.77 \pm 1.48$ vs. $5.7 \pm 1.18, p<0.001)$. Using MK-801 before administration of $\mathrm{CE}$ had no significant effect on rotarod balance time but picrotoxin injection could reduce the balance time significantly compared to CE-treated group and neuropathy signs returned $(2.53 \pm 0.45$ vs. $24.77 \pm 1.48$, $p<0.001$, Fig. 4b).

\section{Foot fault counting test}

As it was predicted, the foot fault number increased significantly in the pyridoxine group in comparison with control group $(7.75 \pm 1.14$ vs. $1.12 \pm 0.79, p<0.001$, Fig. 5a). The pyridoxine-treated rats were unable to move on bars and fell down between the grids. According to Fig. 5b, administration of $50 \mathrm{mg} / \mathrm{kg}$ of CE improved neuropathy signs and reduced significantly the fault number compared to the saline-treated group $(3.14 \pm 0.75$ vs. $7.25 \pm 1.32, p<0.01)$. Administration of MK-801 before $\mathrm{CE}$ had no affect on foot fault number in comparison with CE group. In the picrotoxin-treated group,
Fig. 3 Images of the rats suffering from neuropathy induced by pyridoxine toxicity for 14 days. They were unable to stand or walk on all four paws

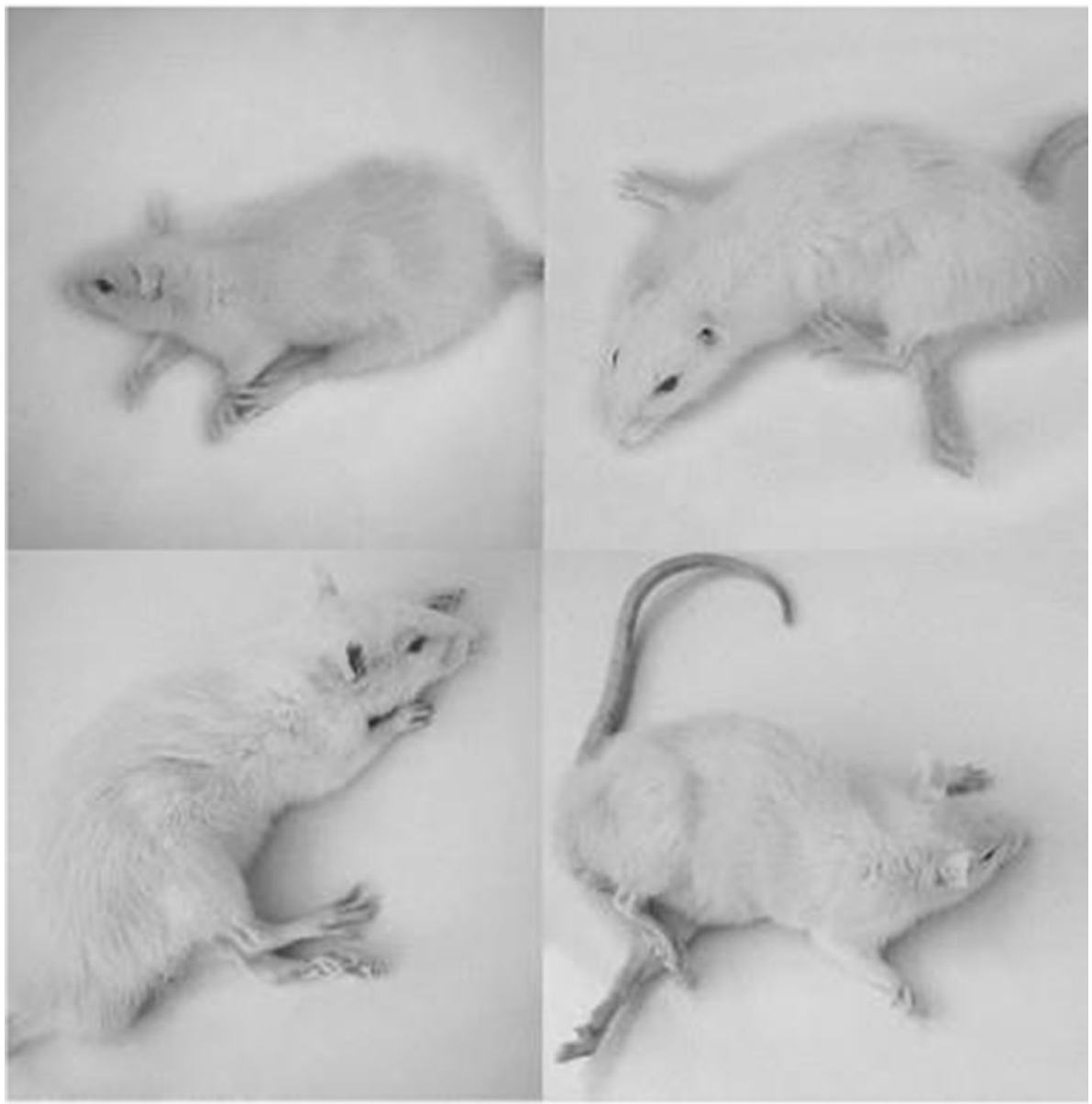



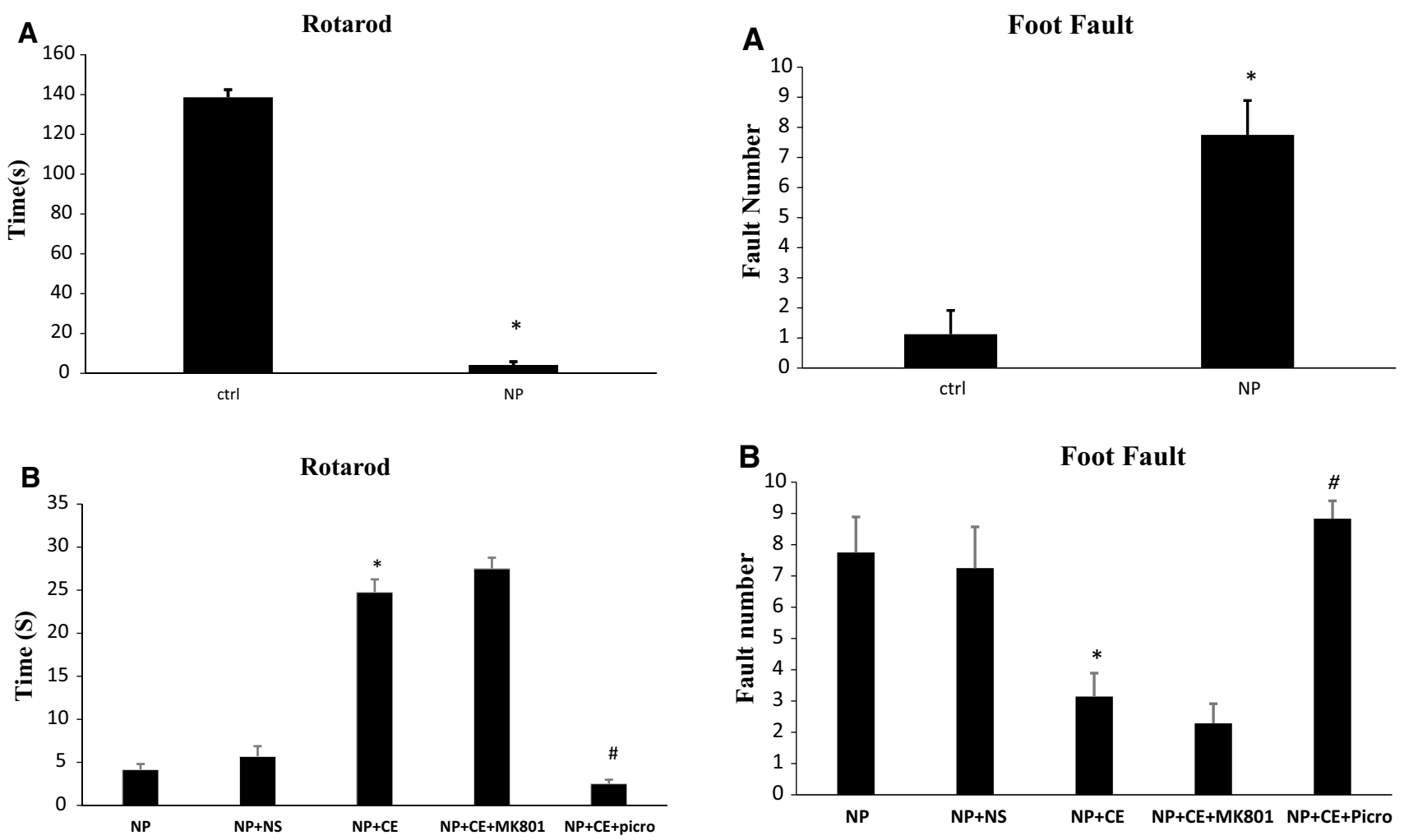

Fig. 4 Pyridoxine-induced neuropathy and investigation of the role and probable mechanism of $\mathrm{CE}$ in the rotarod test. a High dose of pyridoxine significantly decreased rotarod time compared to control (ctrl) group $\left({ }^{*} p<0.001\right)$. b In the treatment group, CE increased rotarod time in comparison with saline group (NS) $(* p<0.001)$. MK-801 had no significant effect while picrotoxin (picro) decreased rotarod time compared to the CE group $(\# p<0.001)$. Data are shown as mean $\pm \operatorname{SEM}(N=7$ per group)

fault number increased significantly compared to the CE group $(8.83 \pm 0.57$ vs. $3.14 \pm 0.75, p<0.001)$.

\section{Hot plate test}

The reaction time of animals in the pyridoxine-receiving group increased in comparison with the control group regarding the hot plate test $(7.43 \pm 3.61$ vs. $3.61 \pm 0.28$, $p<0.001$, Fig. 6a). Furthermore, administration of $50 \mathrm{mg} /$ $\mathrm{kg} /$ i.p. of CE significantly decreased the reaction time compared to the saline-treated group $(4.81 \pm 0.49$ vs. $7.22 \pm 0.66, p<0.05)$. No significant change was found in the MK-801 pre-treated group compared to the CEtreated group but the picrotoxin-recipient group showed

Fig. 5 Pyridoxine-induced neuropathy and investigation of the role and probable mechanism of CE in foot fault test. a Foot fault increased in the pyridoxine group compared to the control (ctrl) group $(* p<0.001)$. b CE had a beneficial effect to reduce fault number compared to saline (NS)-treated group $(* p<0.01)$. The effect of MK-801 was not significant compared to the CE-treated group while picrotoxin (picro) could increase the number of foot fault in comparison with CE-treated group significantly $(\# p<0.001)$. Data are shown as the mean $\pm \operatorname{SEM}(N=7$ per group)

a significant increase in reaction time in comparison with the CE-treated group $(10.3 \pm 0.38$ vs. $4.81 \pm 0.49$, $p<0.001$, Fig. 6b).

\section{Histological and quantitative analysis of DRG neurons}

According to the observation of DRG neurons in $\mathrm{H} \& \mathrm{E}$ staining, the cells were divided into two groups (A-cells and B-cells) based on size, appearance, and histochemical reactions. Type A cells have a large size and their cytoplasm has occupied the entire cell with a light background. The nucleus of the neurons is generally lightly stained, usually with one large and less intensely stained central nucleolus. In contrast, type B cells have a dark cytoplasm that is more homogeneous and intensely stained than A-cells. Additionally, type B is usually 

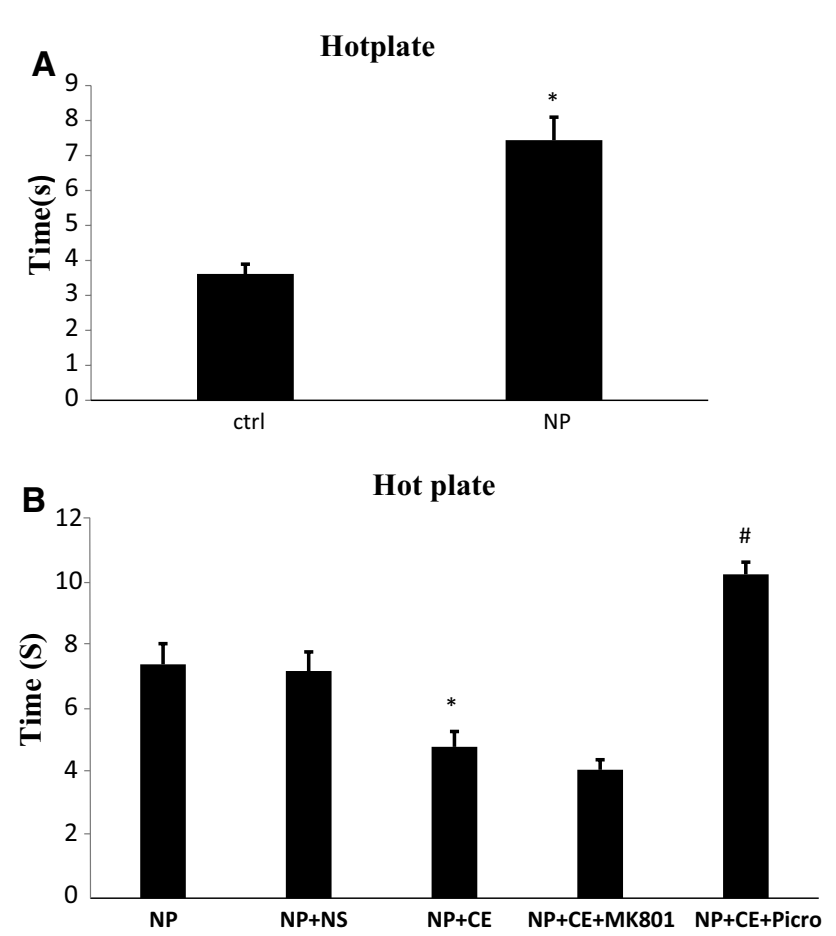

Fig. 6 Pyridoxine-induced neuropathy and investigation of the role and probable mechanism of $\mathrm{CE}$ in hot plate test. a Reaction time to heat increased in pyridoxine group compared with the control (ctrl) group. ${ }^{*} p<0.001$. b The rats receiving CE decreased reaction time in comparison with saline (NS) group $(* p<0.05)$. Picrotoxin (picro) treatment group increased the duration of response to heat but Mk-801 had no significant effect $(\# p<0.001)$. Data are shown as the mean $\pm \operatorname{SEM}(N=7$ per group $)$

smaller and vacuolation is also evident in them. The nucleus is characterized by more basophilic and many smaller chromatin condensations. Based on our observations, the number of B-cells was more abundant in the neuropathy group compared to the control group. Furthermore, the degenerating neurons showed morphological characteristic of apoptosis, which was not present in the control group. Observation of the DRG neurons in the chicory-treated group clarified that the number of the A-cells increased and CE could exert a significant protective effect by reducing the B-cells. Vacuolation was also reduced in the cells dramatically (Fig. 7).

\section{Histological and quantitative analysis of sciatic nerve}

Cross and longitudinal sections of the sciatic nerve and $\mathrm{H} \& \mathrm{E}$ staining showed that in the control rats, the myelinated nerve fibers were the same in size, density, and form. In the neuropathy group, endoneurium areas were developed and separated from each other and visible symptoms of axonal atrophy were apparent. Furthermore, degenerating fiber profiles including crumbled and irregularly shaped myelin sheaths was seen and the number of fibers decreased. CE-treated animals showed significant improvements in the number of sciatic neural fibers compared with the neuropathy group (Fig. 8).

\section{Measurement of TNF- $a$ level in rat DRG and sciatic nerve}

Figure 9 represents the expression of TNF- $\alpha$ in the DRG and sciatic nerve of control, pyridoxine, and CE-treated groups. Also, the effect of pre-treatment with MK-801 and picrotoxin in the CE-treated group is shown. After induction of neuropathy, a rapid increase in TNF- $\alpha$ levels was observed in both DRG $(101.56 \pm 0.91$ vs. $40.66 \pm 1.42, p<0.001)$ and sciatic nerve $(79.45 \pm 0.75$ vs. $29.41 \pm 1.11, p<0.001)$ in comparison with control rats. Ten days after CE injection, the content of TNF- $\alpha$ in the CE-treated group was reduced compared to the NP group in DRG $(62.51 \pm 1.03$ vs. $101.56 \pm 0.9, p<0.001)$ and sciatic nerve $(51.59 \pm 0.96$ vs. $79.45 \pm 0.75, p<0.001)$. The rats that were pre-treated with picrotoxin before $\mathrm{CE}$, unlike the MK-801 pre-treated group, displayed significantly higher TNF- $\alpha$ expression than the CE-alone group in both their DRG $(105.26 \pm 1.15$ vs. $62.51 \pm 1.03, p<0.001)$ and sciatic nerve $(71.21 \pm 1.24$ vs. $51.59 \pm 0.96, p<0.01)$.

\section{Discussion}

The data collected through rotarod, foot fault, and hot plate tests, histological analysis, and measurement of levels of TNF- $\alpha$ showed that injection of CE with a positive effect could decrease the signs of neuropathy to some extent in all three behavioral tests and these changes were also evident in histological analysis and TNF- $\alpha$ content levels in DRG and sciatic nerve of neuropathic rats. Administration of GABA antagonist (picrotoxin) before CE intensified the severity of neuropathy but glutamate antagonist (MK-801) had no significant effect on neuropathy.

Based on the studies, pyridoxine intoxication induces sensory and peripheral neuropathy in rats and eventually also causes some lack of balance, decreased heat sensitivity, motor ataxia, and weight loss. A high dose of pyridoxine causes neuropathy by DRG neuronal necrosis and degradation of peripheral and central sensory fibers. Microscopic observations show that the primary site of injury in pyridoxine neurotoxicity is in the soma of 

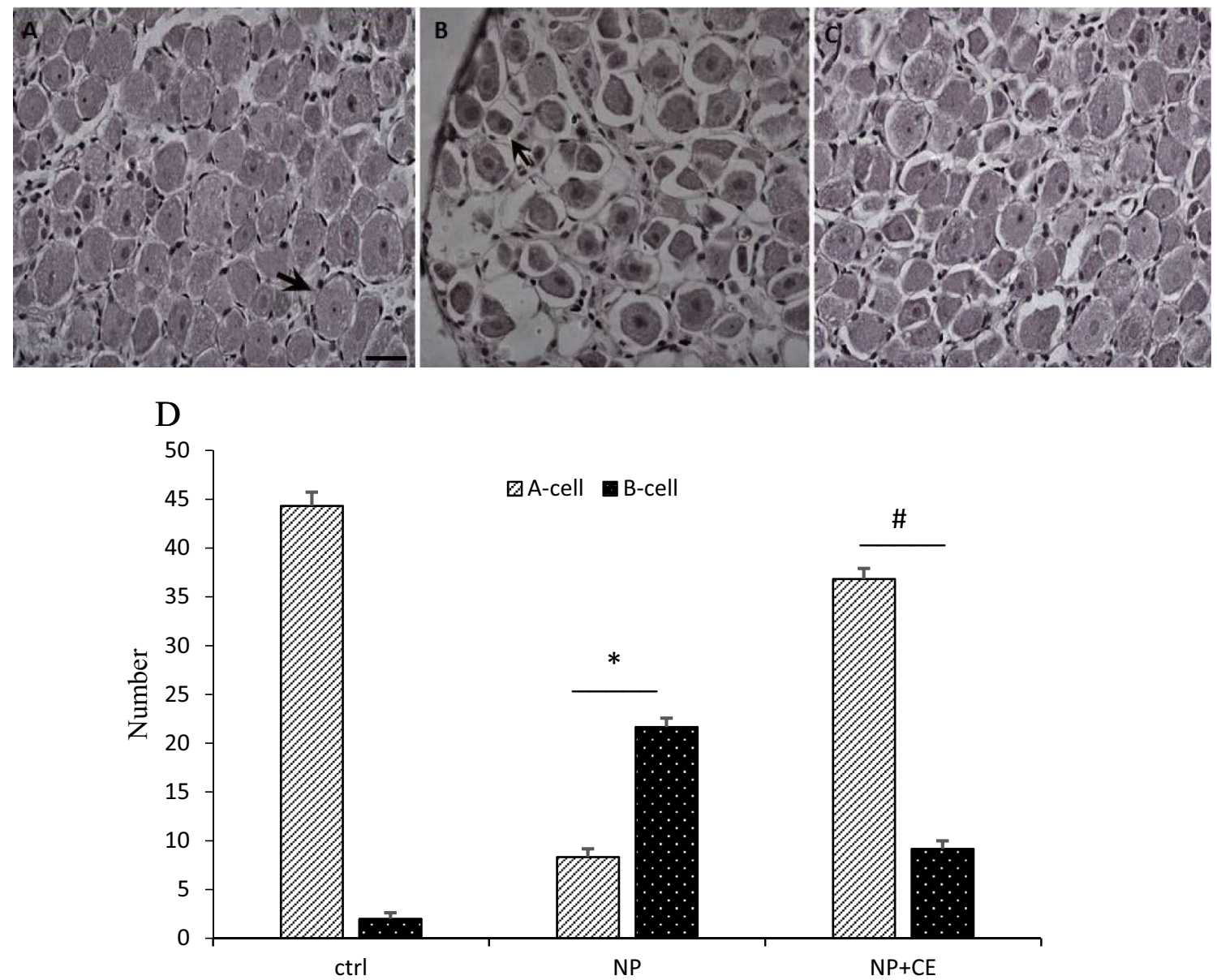

Fig. 7 Cross sections of dorsal root ganglion neurons (DRG) from control (a), rats received a high dose of pyridoxine (b), rats treated with CE (c), and the chart of DRG cells number (d). There are two types of cells in DRG neurons: A type or B type. Type A cells have a large size and less intensely central nucleolus (arrow in a). B cells are characterized by a nucleus containing basophilic and many smaller chromatin condensations (arrow in b). The result of the cell count

neurons of the DRG, which leads to destruction of the axonal integrity of the long myelinated fibers in the sciatic nerve. In general, toxicity of pyridoxine leads to remarkable sciatic nerve axonopathy and an increased endoneurial area, which is probably related to axonal degeneration together with the increase of the number of small diameter fibers. Furthermore, initial observations suggested that with the destruction of DRG neurons, Nissl bodies, as well as many cavities, develop in the cytoplasm, which in turn reduces cytoplasm volume [27]. Necrotic cell death stimulates inflammatory responses and can lead to the release of pro-inflammatory mediators and therefore might worsen the symptoms [29]. showed that the number of A-cells are more abundant than B-cells in the control group. However, in the neuropathic group compared to the control, the number of B-cells increased significantly $\left({ }^{*} p<0.001\right)$. $\mathrm{CE}$ treatment abolished the effect of pyridoxine toxicity and led to an increase in the number of A-cells $(\# p<0.001)$ ( $\mathrm{H} \& \mathrm{E}$ magnification $200 \times$, scale bar $50 \mu \mathrm{m}$ )

This type of neuropathy is similar to chemotherapyinduced neuropathy (such as cisplatin and oxaliplatin therapy) [30] and metabolic neuropathy (diabetes-associated neuropathy) $[13,31]$ considering its symptoms and site of injury (DRG neurons).

Several studies have shown that medicinal plants can be used to treat these models of neuropathy [32-34]. Based on our results, CE has had an improving effect on the rotarod and foot fault tests performance. Moreover, sensory reaction and the reaction time were reduced in the hotplate test. Also, the histological analysis confirmed the CE beneficial effect on DRG and sciatic nerve injuries. Measuring the level of TNF- $\alpha$ in two tissues revealed that CE can 

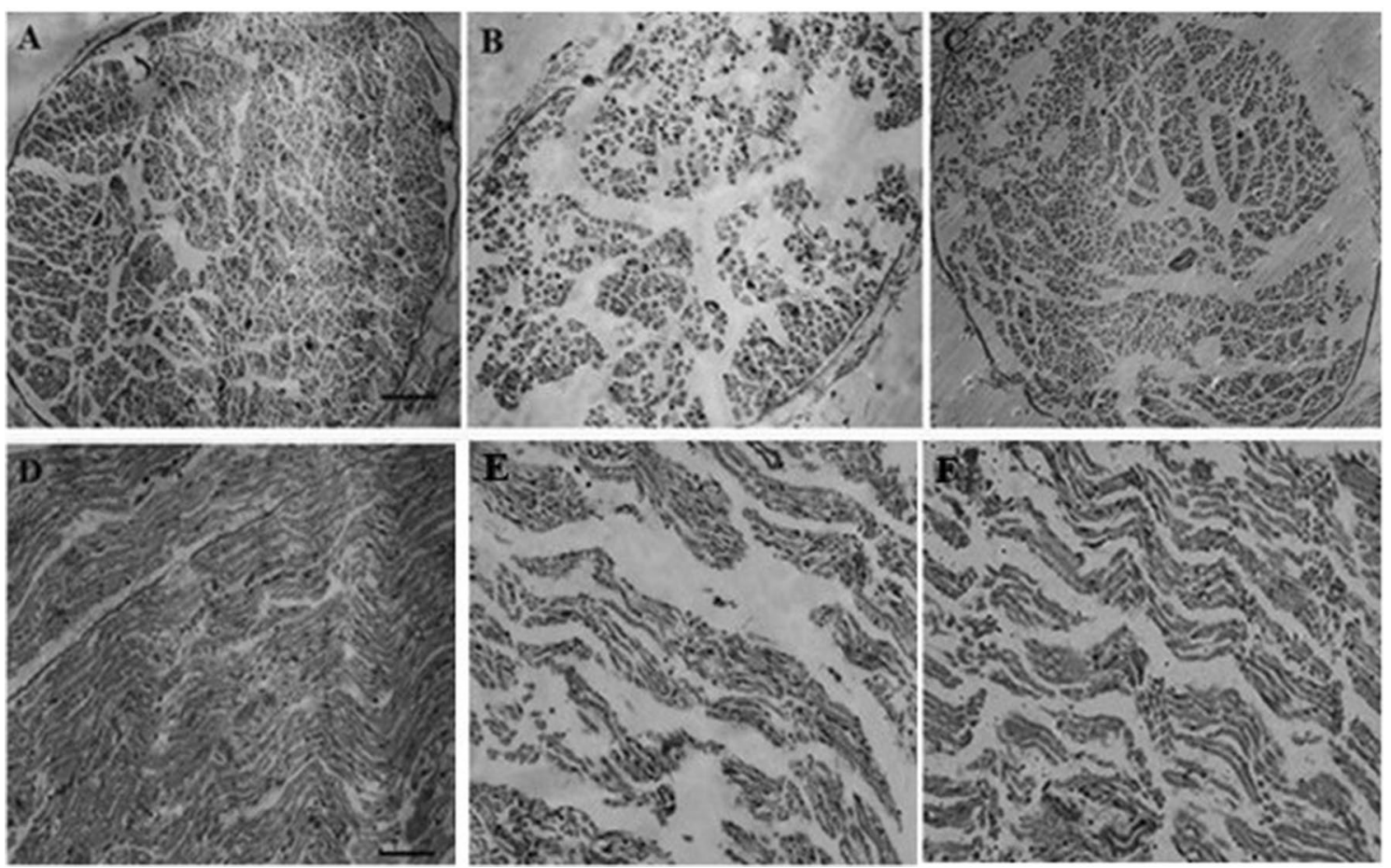

Fig. 8 Cross $(\mathbf{a}-\mathbf{c})$ and longitudinal $(\mathbf{d}-\mathbf{f})$ sections of sciatic nerve from control, neuropathy, and CE-treated groups, respectively. In the control group, the number of nerve fibers in both cross and longitudinal sections is greater than the neuropathy group. In chicory-treated

significantly reduce TNF- $\alpha$ levels in rats. It was shown that after peripheral nervous injury, endogenous TNF- $\alpha$ dramatically increases in medium to large size of DRG neurons in sciatic nerves, and this may increase TNF- $\alpha$ in the muscle and contribute to the progression of neuropathic pain [35].

It seems that the mechanism of $\mathrm{CE}$ is through the GABAergic system. The function of GABAergic neurons in peripheral sensory ganglia can modulate transmission of pain-related signals from the peripheral sensory nerves to the CNS [36]. Based on previous studies, chicory root extract can control inflammation in two ways: (1) inhibition of TNF- $\alpha$ and in consequence the reduction of COX2 , and (2) direct inhibition of COX-2 activity and downregulation of COX enzyme [37]. In our study also, TNF- $\alpha$ levels decreased with chicory injection in neuropathic rats. Studies on the effects of CE have indicated that compounds of chicory root such as glycosides and flavonoids can modulate the function of ionotrophic GABA receptor. In other words, they elevate the expression level of GABA receptor and especially increase GABA type A receptors [38, 39]. On the other hand, it appears that glycosides, sterols, polyphenols, flavonoids, tannins, and terpenoids have antiinflammatory activity by reducing inflammatory mediators rats, the number of nerve fibers increased and also endoneurium areas appeared smaller than the pyridoxine-treated animals. (H\&E magnification $100 \times(\mathbf{a}-\mathbf{c})$, scale bar $100 \mu \mathrm{m}$; H\&E magnification: 200× (d-f), scale bar $50 \mu \mathrm{m})$

including TNF- $\alpha$, prostaglandins, NO, IL-6, and IL-1 [25]. Moreover, triterpenoids and sesquiterpene lactone of chicory root have the potential to inhibit COX-2 and so in addition to control inflammation, they can increase GABAergic neurons $[15,37]$.

In the current study, we used GABA antagonist (picrotoxin) before $\mathrm{CE}$ to investigate the mechanism of ameliorative effect of the extract. The results showed that pretreatment with picrotoxin intensified neuropathic signs in all three behavioral tests and increased TNF- $\alpha$ level. It has previously been shown that GABA and $\mathrm{GABA}_{\mathrm{A}}$ receptor agonist reduces inflammatory cytokines and hence can modulate signs of inflammation in the nervous system [40]. Injection of picrotoxin and inhibition of GABA receptors may increase the damage of the DRG neurons and the sciatic nerve. This increases the level of TNF- $\alpha$ and exacerbates the peripheral neuropathy. Thus, it is highly probable that increasing $\mathrm{GABA}_{\mathrm{A}}$ receptors activity especially in DRG neurons and sciatic nerve by $\mathrm{CE}$ and reducing TNF- $\alpha$ level can induce inhibitory effect on the symptoms of pyridoxine toxicity and peripheral neuropathy (Fig. 10). 

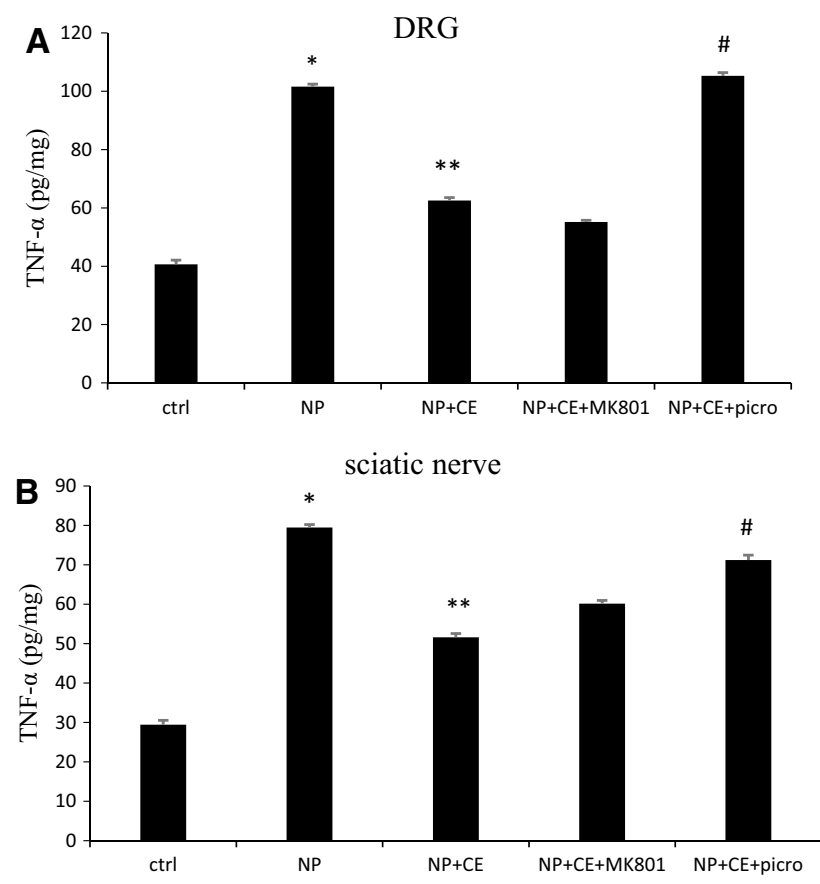

Fig. 9 Measurement of TNF- $\alpha$ level in the DRG (a) and sciatic nerve (b). Protein levels were determined by ELISA. Data are shown as the mean $\pm \operatorname{SEM}(N=5$ per group). $* p<0.001 \mathrm{NP}$ vs. ctrl (a and b); $* * p<0.001 \mathrm{NP}+\mathrm{CE}$ vs. NP (a and b); $\# p<0.001$ (a) and $p<0.01$,

(b) $\mathrm{NP}+\mathrm{CE}+$ picro vs. $\mathrm{NP}+\mathrm{CE}$ (a and $\mathbf{b})$
Despite our hypothesis about the role of glutamate on pyridoxine-induced neuropathy, our results did not show a significant effect on signs of neuropathy in comparison with CE in all tests. In this study, MK-801 was used as a non-competitive NMDA receptor antagonist. Based on investigations, there are three groups of metabotropic glutamate receptors. Group 1, increases NMDA inotropic receptor activity and hence promotes the neuronal injury while the other two groups decrease NMDA receptor activity and cause neuroprotective effects [41-43]. Previous studies had clarified that glutamate can act dose-dependently, meaning that different doses lead to different effects. Actually, it might be true that high doses of glutamate influence group 1 receptor and low doses of it are able to act on the other two groups [44]. According to Mahiuddin Ahmed et al., MK-801 also acts dose-dependently and has both neuroprotective and neurotoxic effects, depending on its dose in rat brain regions [45].

Although it was shown that MK-801 can reduce the expression of TNF- $\alpha$ in DRG neuron and sciatic nerves [46], our results showed no significant difference between TNF- $\alpha$ level in the extract and MK-801 groups. According to the above description, due to the characteristics of MK-801 and glutamate receptors, it seems that CE cannot act on glutamate receptor. It seems that perhaps symptoms of neuropathy were significantly accelerated by selecting a higher dose of MK-801.
Fig. 10 Diagram of chicory mechanism stimulation and inhibition

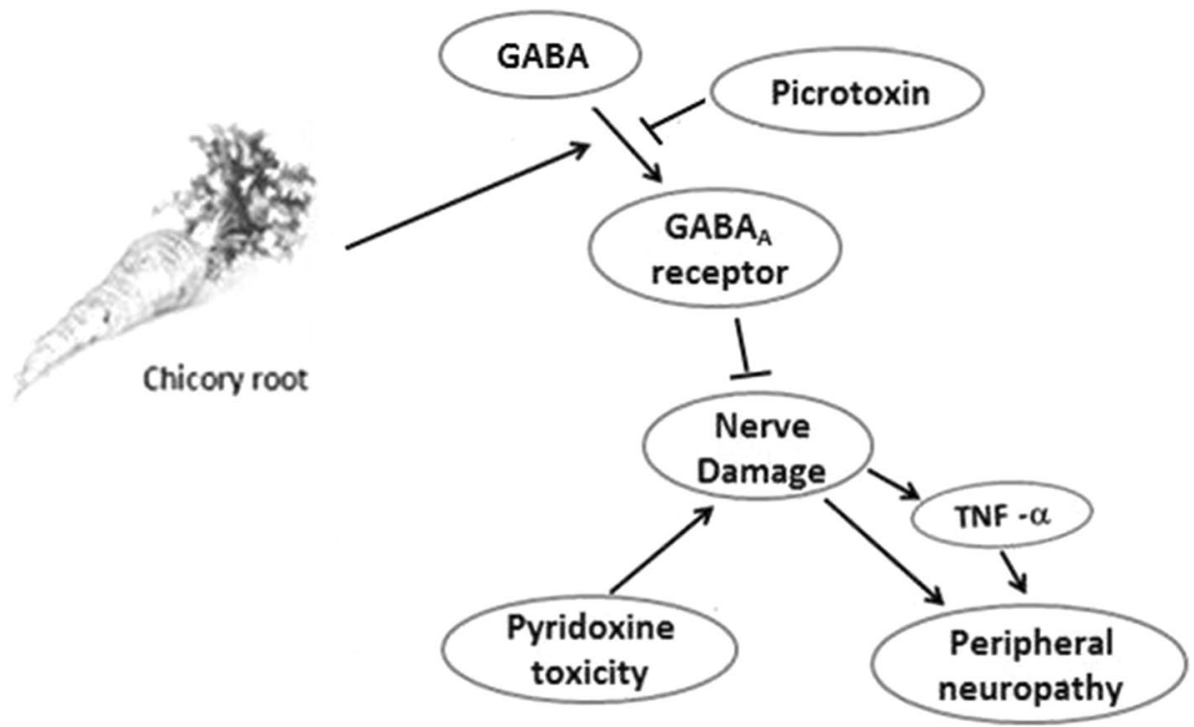




\section{Conclusions}

Our results showed beneficial effects of $\mathrm{CE}$ on pyridoxineinduced peripheral neuropathy. Modulating of the GABAergic neurotransmitter-mediated reduction of TNF- $\alpha$ may be involved in the anti-neurotoxic and neuroprotective effect of chicory.

Acknowledgements We acknowledge and appreciate Dr. Mostafa Golshekan for assisting with the GC-MS and Mostafa Haghiri for help with editing and improving the manuscript.

\section{Compliance with ethical standards}

Conflict of interest All named authors hereby declare that they have no conflicts of interest to disclose.

\section{References}

1. Costigan M, Scholz J, Woolf CJ (2009) Neuropathic pain: a maladaptive response of the nervous system to damage. Annu Rev Neurosci 32:1-32

2. Cojocaru IM et al (2014) Peripheral nervous system manifestations in systemic autoimmune diseases. Mædica 9(3):289

3. Azhary $\mathrm{H}$ et al (2010) Peripheral neuropathy: differential diagnosis and management. Am Fam Physician 81(7):887-892

4. Kelly JJ, Karcher DS (2005) Lymphoma and peripheral neuropathy: a clinical review. Muscle Nerve 31(3):301-313

5. Mooney S et al (2009) Vitamin B6: a long known compound of surprising complexity. Molecules 14(1):329-351

6. Chaudary AN, Porter-Blake A, Holford P (2003) Indices of pyridoxine levels on symptoms associated with toxicity: a retrospective study. J Orthomol Med 18(2):65-76

7. Levine S, Saltzman A (2004) Pyridoxine (vitamin B6) neurotoxicity: enhancement by protein-deficient diet. J Appl Toxicol 24(6):497-500

8. Dalton K, Dalton MJT (1987) Characteristics of pyridoxine overdose neuropathy syndrome. Acta Neurol Scand 76(1):8-11

9. Callizot N, Warter J-M, Poindron P (2001) Pyridoxine-induced neuropathy in rats: a sensory neuropathy that responds to 4-methylcatechol. Neurobiol Dis 8(4):626-635

10. Ollivier-Lanvin $\mathrm{K}$ et al (2010) Proprioceptive neuropathy affects normalization of the H-reflex by exercise after spinal cord injury. Exp Neurol 221(1):198-205

11. Azevedo MI et al (2013) The antioxidant effects of the flavonoids rutin and quercetin inhibit oxaliplatin-induced chronic painful peripheral neuropathy. Mol Pain 9(1):53

12. Areti A et al (2014) Oxidative stress and nerve damage: role in chemotherapy induced peripheral neuropathy. Redox Biol 2:289-295

13. Schmeichel AM, Schmelzer JD, Low PA (2003) Oxidative injury and apoptosis of dorsal root ganglion neurons in chronic experimental diabetic neuropathy. Diabetes 52(1):165-171

14. Ma W, Quirion R (2008) Does COX2-dependent PGE2 play a role in neuropathic pain? Neurosci Lett 437(3):165-169

15. Coballase-Urrutia E et al (2017) Biochemical and molecular modulation of CCl4-induced peripheral and central damage by Tilia americana var. mexicana extracts. Saudi Pharm J 25(3):319-331

16. Nutt D (2006) GABA A receptors: subtypes, regional distribution, and function. J Clin Sleep Med 2(02):S7-S11
17. Moore KA et al (2002) Partial peripheral nerve injury promotes a selective loss of GABAergic inhibition in the superficial dorsal horn of the spinal cord. J Neurosci 22(15):6724-6731

18. Karim F, Bhave G, Gereau IV R (2001) Metabotropic glutamate receptors on peripheral sensory neuron terminals as targets for the development of novel analgesics. Mol Psychiatry 6:615-617

19. Parfenova $\mathrm{H}$ et al (2006) Glutamate induces oxidative stress and apoptosis in cerebral vascular endothelial cells: contributions of HO-1 and HO-2 to cytoprotection. Am J Physiol Cell Physiol 290(5):C1399-C1410

20. Street RA, Sidana J, Prinsloo G (2013) Cichorium intybus: traditional uses, phytochemistry, pharmacology, and toxicology. Evid Based Complement Altern Med 2013:1-13

21. Shad M et al (2013) Determination of some biochemicals, phytochemicals and antioxidant properties of different parts of Cichorium intybus L.: a comparative study. J Anim Plant Sci 23(4):1060-1066

22. Molan AL et al (2003) Effects of condensed tannins and crude sesquiterpene lactones extracted from chicory on the motility of larvae of deer lungworm and gastrointestinal nematodes. Parasitol Int 52(3):209-218

23. Nishimura $\mathrm{M}$ et al (2015) Effects of the extract from roasted chicory (Cichorium intybus L.) root containing inulin-type fructans on blood glucose, lipid metabolism, and fecal properties. J tradit Complement Med 5(3):161-167

24. Zafar R, Ali SM (1998) Anti-hepatotoxic effects of root and root callus extracts of Cichorium intybus L. J Ethnopharmacol 63(3):227-231

25. Rizvi W et al (2014) Anti-inflammatory activity of roots of Cichorium intybus due to its inhibitory effect on various cytokines and antioxidant activity. Anc Sci Life 34(1):44

26. Stein S (1990) Mass spectral database and software. Version 3.02. National Institute of Standards and Technology (NIST), Gaithersburg

27. Perry TA et al (2004) Pyridoxine-induced toxicity in rats: a stereological quantification of the sensory neuropathy. Exp Neurol 190(1):133-144

28. Kenward MG (1987) A method for comparing proiles of repeated measurements. Appl Stat 36(3):296-308

29. Rock KL, Kono H (2008) The inflammatory response to cell death. Annu Rev Pathol Mech Dis 3:99-126

30. Lees JG et al (2017) Immune-mediated processes implicated in chemotherapy-induced peripheral neuropathy. Eur J Cancer 73:22-29

31. Rasoulian B et al (2018) Neuroprotective and antinociceptive efects of rosemary (Rosmarinus officinalis L.) extract in rats with painful diabetic neuropathy. J Physiol Sci 61(10):1-8

32. Lee JS et al (2012) Effect of green tea extracts on oxaliplatininduced peripheral neuropathy in rats. BMC Complement Altern Med 12(1):124

33. Chen $\mathrm{W}$ et al (2013) Chinese herbal medicine for diabetic peripheral neuropathy. Cochrane Libr 10(1):1-137

34. Lee G, Kim SK (2016) Therapeutic effects of phytochemicals and medicinal herbs on chemotherapy-induced peripheral neuropathy. Molecules 21(9):1252

35. Schäfers M et al (2002) Anterograde transport of tumor necrosis factor- $\alpha$ in the intact and injured rat sciatic nerve. J Neurosci 22(2):536-545

36. Du X et al (2017) Local GABAergic signaling within sensory ganglia controls peripheral nociceptive transmission. J Clin Investig 127(5):1741-1756

37. Cavin $\mathrm{C}$ et al (2005) Inhibition of the expression and activity of cyclooxygenase- 2 by chicory extract. Biochem Biophys Res Commun 327(3):742-749

38. Hanrahan JR, Chebib M, Johnston GA (2011) Flavonoid modulation of GABAA receptors. Br J Pharmacol 163(2):234-245 
39. Strommer B et al (2014) A cycloartane glycoside derived from Actaea racemosa L. modulates GABAA receptors and induces pronounced sedation in mice. J Pharmacol Exp Ther 351(2):234-242

40. Bhat $\mathrm{R}$ et al (2010) Inhibitory role for GABA in autoimmune inflammation. Proc Natl Acad Sci 107(6):2580-2585

41. Swanson CJ et al (2005) Metabotropic glutamate receptors as novel targets for anxiety and stress disorders. Nat Rev Drug Dis$\operatorname{cov} 4(2): 131$

42. Lea PM IV et al (2002) Neuronal and glial mGluR5 modulation prevents stretch-induced enhancement of NMDA receptor current. Pharmacol Biochem Behav 73(2):287-298

43. Skeberdis VA et al (2001) mGluR1-mediated potentiation of NMDA receptors involves a rise in intracellular calcium and activation of protein kinase C. Neuropharmacology 40(7):856-865
44. Arkaravichien T et al (2003) Dose-dependent effects of glutamate in pyridoxine-induced neuropathy. Food Chem Toxicol 41(10):1375-1380

45. Ahmed MM et al (2003) Dose-dependent effect of MK-801 on the levels of neuropeptides processing enzymes in rat brain regions. Neurosci Res 47(2):177-189

46. Leonoudakis D et al (2004) TNF $\alpha$-induced AMPA-receptor trafficking in CNS neurons; relevance to excitotoxicity? Neuron Glia Biol 1(3):263-273

Publisher's Note Springer Nature remains neutral with regard to jurisdictional claims in published maps and institutional affiliations. 